\title{
Calculating the activation energies of nickel, manufactured using 3D printing technology, with multichannel hydrogen diffusion model
}

\author{
Petr Zumberov ${ }^{1,2, *}$, Sergey Kolesov ${ }^{1}$, Vladimir Polyansky ${ }^{2,1}$, and Evgeniy Varshavchik ${ }^{1,2}$ \\ ${ }^{1}$ Peter the Great St. Petersburg Polytechnic University, St. Petersburg, Russia \\ ${ }^{2}$ Institute for Problems in Mechanical Engineering of the Russian Academy of Sciences, St. Petersburg, Russia
}

\begin{abstract}
In modern world more and more materials with extreme properties are being used: high alloys, high-entropy alloys, nanostructured materials, etc. The extreme properties of these materials make them especially sensitive to hydrogen diffusion. Hydrogen can severely impair their properties and cause failures in structures and machines the material is used in. Nowadays, when structures and components are becoming increasingly complex, the use of 3D printing technology is becoming more widespread. Components made using 3D printing technologies are usually layered, which increase the amount of hydrogen that can diffuse into the material. The amount of hydrogen concentration in 3D printed nickel samples has been determined using vacuum heating method in hydrogen analyzer AV-1. The samples were held at different constant temperatures and the total amount of hydrogen extracted at those temperatures was calculated. A mathematic model was developed to evaluate the amount of hydrogen extracted at a given temperature. The evaluation was then compared to the experiment results, and the validity of the mathematical model and the selected hydrogen activation energies was verified.
\end{abstract}

\section{Introduction}

Additive technologies allow to create complex metal parts without using too much special equipment. Another obvious advantage is the ability to alter the composition of a metal and its microstructure directly in the process of production of a part cf. [1-4].

Along with that, there is a few problems.

* Authors of [5] reported that fatigue cracks initiating at pores close to the surface for additive processed $\mathrm{Ti}$ $6 \mathrm{Al}-4 \mathrm{~V}$. Porosity is attributed to the absorption of $\mathrm{N}, \mathrm{O}$, and $\mathrm{H}$ in the molten weld pool; this then nucleates at the solid-liquid interface during solidification. The fatigue life of most of the specimens exceeded the baseline; however, a small number failed at a very early stage of testing.

* In [6] discussed that porosity is a common concern in metal AM. Other defects (residual stress, delamination, cracking, swelling, etc.) are unique to welding or metal AM.

Among the gasses, adsorbed on the particles of the powder, water vapor represents a major portion of them. Dissociation of this vapor during the welding of the powder can produce significant amounts of hydrogen $\mathrm{cf}$. [5].

Hydrogen also plays a key role in corrosion processes cf. [7-9] and in interactions between a metal and an environment cf. $[10,11]$.

\footnotetext{
*Corresponding author: peterz.98@yandex.ru
}

For engineering and scientific purposes, understanding the mechanism of the interaction of hydrogen with defects and structural features of materials is a relevant problem cf. [12]. However, we only know of a virtually single fundamental study on this topic [13]. This study describes experiments provided for a titanium alloy $\mathrm{Ti}-6 \mathrm{Al}-4 \mathrm{~V}$.

Nickel and its alloys are of utmost importance in modern technologies cf. [14]. Studying the interaction of additive processed nickel with hydrogen is an important problem.

\section{The most common method}

Inside metals hydrogen is located in traps of different nature cf. [15]. The activation energy of hydrogen in the traps characterizes the force of its interaction with the structural elements of a metal and its mobility inside a metal. This is especially important for practical purposes, because hydrogen can locally concentrate, due to high mobility, and serve as the initiator of a failure $\mathrm{cf}$. [16-18].

The standard method for evaluating the binding energies of hydrogen in the traps is the method of thermo-desorption spectra (TDS) cf. $[19,20]$. The method is based on the Kissinger model cf. [21].

Now, virtually all the studies on the interaction of hydrogen with metals are carried out using the method of TDS, including new materials cf. [13]. 
However, this method has serious disadvantages. The main disadvantage of this method is the fact that it does not account for diffusion inside the material. In fact, only surface sorption and desorption can be adequately evaluated using this method [22]. This leads to large variations in activation energy values for the same materials [23-26].

\section{Experimental Technique}

The selected samples were cylindrical $2 \mathrm{~mm}$ in diameter and $10 \mathrm{~mm}$ in height. The samples were manufactured using selective laser melting (SLM) from powdered nickel Alloy 718 / Inconel 718.

The hydrogen charging of the samples was done using cathodic polarization method in $3 \mathrm{M} \mathrm{NaCl}$ solution. The charging current was $0.11 \mathrm{~A}$. The duration of the charging was 125 hours. The duration was selected that the hydrogen would be evenly distributed cf. [27]. According to the data presented in [28], for a sample $8 \mathrm{~mm}$ in diameter, 500 hours were needed for the even distribution. The uniformity of the distribution was not controlled in any other way.

Experiment data was obtained using industrial hydrogen analyser AV-1. The hot vacuum extraction method was used to carry out the measurements cf. [22,23,25].

An increase of the temperature of the extraction system leads to an exponential growth of the background flows of hydrogen. This effect is usually not discussed, however, research shows, that the growth of the background flows can significantly impact the results of the measurements with a constant increase in temperature of a studied sample cf. [24, 29, 30].

The significance of the effect of the background flows can be seen on the extraction curves, shown in Fig.1. The figure shows the dependence of the flow of hydrogen on the time of heating. The curve on the left corresponds to a situation, when a cold steel sample is dropped in the extraction system, preheated to $600{ }^{\circ} \mathrm{C}$. The curve on the right corresponds to an empty extraction system being heated from 600 to $800{ }^{\circ} \mathrm{C}$. Both for the sample and for the empty extraction system the equivalent concentration of hydrogen $C_{\mathrm{H}}$ is indicated.

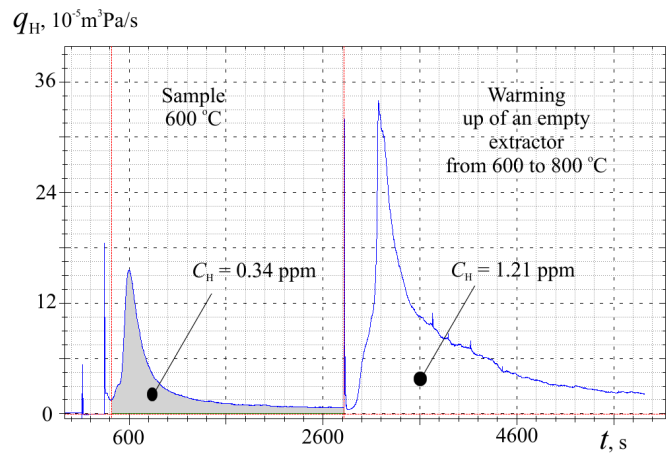

Fig. 1. Extraction curves of the flow of hydrogen $q_{\mathrm{H}}$ from the steel sample at a temperature of $600^{\circ} \mathrm{C}$ (left) and an empty extraction system during heating from 600 to $800^{\circ} \mathrm{C}$ (right). The area under the graphs is proportional to the amount of extracted hydrogen.
With regard to the significant impact of the background flows, we applied a procedure of step increase of the extraction temperature with a constant extraction time on each step.

After the extraction system is prepared (the samples were loaded, the vacuum was pumped) the temperature is set below the temperature usually needed to extract all hydrogen from a sample (usually, it is $150-200^{\circ} \mathrm{C}$ ).

After the background flows settle, the first analysis is performed by the standard procedure of recording the extraction curve and the evaluation of the concentration of hydrogen released at this temperature. Then, the sample is moved to the cold part of the extractor.

The graph, indicating the time dependence on the temperature of the extraction, is shown in Fig.2. The distribution of time on each step is also shown in Fig.2. About 1000 seconds is needed for the extraction system to heat up and the background flows to settle. The sample, dropped into the extraction system, is heated during 3000 seconds.

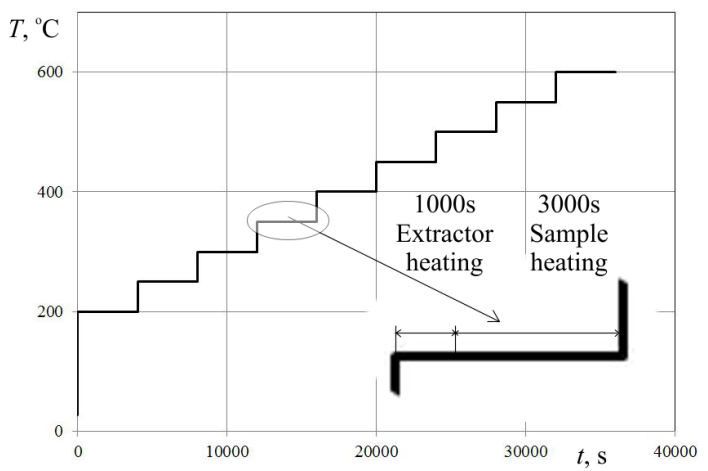

Fig. 2. The time dependence of the temperature of the extraction system.

The extraction temperature (the temperature of the analysing part of the extractor) is increased in steps. After the background flows settle, the same sample is moved back to the analysing part of the extractor. Thus, the extraction curve is recorded multiple times for each sample at different temperatures. The amount of hydrogen extracted at a given temperature is evaluated based on the extraction curve for this temperature.

If the flow of hydrogen does not stop after a significant amount of time during the analysis, the analysis is terminated at a fixed time after the start (in this case, 3000 seconds). Some amount of hydrogen remains in the sample, since it quickly cools after it is removed from the analysing part of the extractor. Discrete thermo-desorption spectra (DTDS), obtained using this method, is a relation between the amount of released hydrogen and temperature. Its main advantage, compared to the standard TDS, is the settling of the background flows, which remain constant during the analysis and can be subtracted from the measured hydrogen flows, without any additional assumptions. A term "isotheral spectra" can be found in literature, which is used to describe the method of step heating of a sample. However, in our case, the change of the extraction temperature is performed without a sample 
inside, that allows to account for the background flows better.

\section{Determination of the activation energies}

\subsection{Mathematical model}

An extraction curve, showing the time dependence of the flow of hydrogen from a sample is recorded on each step. The characteristic shape of the curve is shown in Fig.1.

The integration of the area between the extraction curve and the background flows is done using the AV1 's software. The concentration of hydrogen which was extracted from a sample on each temperature step is calculated, considering the calibration coefficient and the mass of a sample.

The dependence of the concentration of extracted hydrogen on the temperature of a step is a discrete thermo-desorption spectra (DTDS).

Extraction curves for each temperature are modelled. The modelling is based on the mechanism of multichannel diffusion of hydrogen [22]. To calculate the temperature dependence of the diffusion coefficient the Arrhenius law is used.

The calculation of the hydrogen flows and their time integrals, characterizing the integral results of the measurements for each time step, was executed using a Fortran program. The parameters of the sample and the experiment conditions were input into the program. The integration was done with the explicid fourth-order Runge-Kutta method. The activation energy $u_{0}$, diffusion ceconstant $\mathrm{D}_{0}$ and hydrogen concentration $\mathrm{C}_{0}$ were evaluated by fitting to the experiment data.

\subsection{Results}

As a result of the calculations, the graph of DTDS, shown on the Figure 3 was obtained. The graph corresponds to the activation energies presented in the Table 1. On the graph, the experiment and the calculated points of DTDS, which almost fully match, are shown.

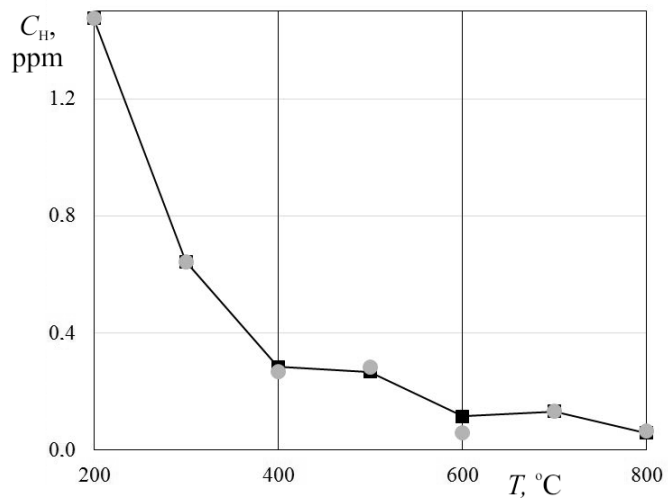

Fig. 3. A graph obtained by the experiment (black) and a graph obtained by the calculation (grey).
Table 1. Activation energies.

\begin{tabular}{|c|c|}
\hline$\#$ & $\begin{array}{c}\text { Activation } \\
\text { energy, eV }\end{array}$ \\
\hline 1 & 0.63 \\
\hline 2 & 0.72 \\
\hline 3 & 0.95 \\
\hline 4 & 1.07 \\
\hline
\end{tabular}

\section{Conclusion}

Almost perfect match of the experiment and calculated DTDS was achieved, which proves the adequacy of the chosen model of multichannel diffusion.

The obtained binding energies are in the range of known values obtained on the base of independent methods. Along with that, the energies are a bit higher than for nickel manufactured using traditional methods.

All this results allows to validate the new method of calculating the binding energies of hydrogen in solids.

The research is carried out under the financial support by Russian Science Foundation, grant 18-19-00160.

\section{References}

1. L. E. Murr, E. Martinez, K. N. Amato, S. M. Gaytan, J. Hernandez, D. A. Ramirez, ... \& R. B. Wicker, J. of Mat. Res. and tech., 1(1), 42-54, (2012)

2. L. E. Murr, E. Martinez, K. N. Amato, S. M. Gaytan, J. Hernandez, D. A. Ramirez, ... \& R. B. Wicker, J. of Mat. Res. and tech., 28(1), 1-14, (2012)

3. D. Ding, Z. Pan, D. Cuiuri, \& H. Li, The Int. J. of Adv. Manuf. Tech., 81(1-4), 465-481, (2015)

4. W. E. Frazier, J. of Mat. Eng. and Perf., 23(6), 1917-1928, (2014)

5. F. Wang, S. Williams, P. Colegrove, and A.A. Antonysamy, Metall. Trans. A., 44A, 968-977, (2013)

6. W. J. Sames, F. A. List, S. Pannala, R. R. Dehoff, \& S. S. Babu, Int. Mat. Rev., 61(5), 315-360, (2016)

7. P. L. Andresen, J. Hickling, A. Ahluwalia, \& J. Wilson. Corr., 64(9), 707-720, (2008)

8. J. E. Draley, \& W. E. Ruther. J. of The Electrochem. S., 104(6), 329-333, (1957)

9. R. E. Ricker, \& D. J. Duquette, Metal. Trans., 19(7), 1775-1783, (1988)

10. R. B. McLellan, \& C. G. Harkins., Mat. Sci. and Eng., 18(1), 5-35, (1975)

11. I. B. Beech, \& J. Sunner, Cur. Opi. in Biotech., 15(3), 181-186, (2004) 
12. G. M. Pressouyre. Metal. and Mat Trans. A, 10(10), 1571-1573, (1979)

13. R. Silverstein, \& D Eliezer, Mat. Char., 144, 297304, (2018)

14. E. O. Ezugwu, Z. M. Wang, \& A. R. Machado, J. of Mat. Proc. Tech., 86(1-3), 1-16, (1999)

15. R. Kirchheim. Acta Metal., 30(6), 1069-1078, (1982)

16. J. Woodtli, \& R. Kieselbach, Eng. Fail. Ana., 7(6), 427-450. (2000)

17. I. M. Robertson, P. Sofronis, A. Nagao, M. L. Martin, S. Wang, D. W. Gross, \& K. E. Nygren, Metal. and Ma. Trans. A, 46(6), 2323-2341, (2015)

18. H. K. Birnbaum, \& P. Sofronis, Mat. Sci. and Eng. A, 176(1-2), 191-202, (1994)

19. R. P. Aveling, U.S. Patent No. 3,068,402. (1962).

20. F. J. Castro, G. Meyer, J. of allo. and Comp., 330 59-63, (2002).

21. H. E. Kissinger, Analy. Chem., 29. 1702-1706, (1957)

22. A. M. Polyanskiy, V. A. Polyanskiy, Y. A. Yakovlev, Int. J. of Hydr. Ener., 39 30, 1738117390, (2014)

23. D. Y. Andronov, D. G. Arseniev, A.. M. Polyanskiy, Int. J. of Hydr. Ener., 42 1, 699-710, (2017)

24. D. G., Arseniev, A.K. Belyaev, V.A. Polyanskiy, Dyn. Pro. in Gen. Cont. and Stru., 37-61, (2019).

25. A. K. Belyaev, A. M. Polyanskiy, V. A. Polyanskiy, Int. J. of Hydr. Ener., 41 20, 8627-8634, (2016)

26. A. Kuduzovic, M.C. Poletti, C. Sommitsch, M. Domankova, S. Mitsche, R. Kienreich Mater. Sci. Eng. 590 66-73 (2014)

27. E. L. Alekseeva, A. K. Belyaev, A. S. Zegzhda, Diagn., Res. and Mech. of mat. and struc., 3, 43-56, (2018)

28. A. Martinsson, R. Sandström, J. of Mat. Sci., 47 19, 6768-6776, (2012)

29. C.V. Tapia-Bastidas, A. Atrens, E. MacA. Gray International Journal of Hydrogen Energy 4315 7600-7617 (2018)

30. N.H. Nickel, K. Brendel, R. Saleh, Physica status solidi, 15 1154-1168 (2004). 